\title{
Drug Response Predictor
}

National Cancer Institute

\section{Source}

National Cancer Institute. Drug Response Predictor. NCI Thesaurus. Code C159906.

A proprietary screening platform that utilizes messenger RNA gene expression

signatures from patient biopsies to identify patients with a high likelihood of responding to specific cancer-fighting therapies. 